\title{
Lidil
}

Revue de linguistique et de didactique des langues

\section{Phraséologie et formules rituelles dans le discours politique, l'expérimentation en lexicométrie}

Lexicometrical Experiment about Phraseology and Ritual Phrases in Political Discours

Jean-Marc Leblanc

\section{OpenEdition}

\section{Journals}

Édition électronique

URL : http://journals.openedition.org/lidil/3930

DOI : $10.4000 /$ lidil.3930

ISSN : $1960-6052$

Éditeur

UGA Éditions/Université Grenoble Alpes

Édition imprimée

Date de publication : 30 mai 2016

Pagination : 43-69

ISBN : 978-2-84310-326-1

ISSN : $1146-6480$

\section{Référence électronique}

Jean-Marc Leblanc, « Phraséologie et formules rituelles dans le discours politique, l'expérimentation en lexicométrie », Lidil [En ligne], 53 | 2016, mis en ligne le 01 janvier 2017, consulté le 29 octobre 2020. URL : http://journals.openedition.org/lidil/3930 ; DOI : https://doi.org/10.4000/lidil.3930

(C) Lidil 


\title{
Phraséologie et formules rituelles dans le discours politique, l'expérimentation en lexicométrie
}

\author{
Jean-Marc Leblanc*
}

\begin{abstract}
RÉSUMÉ
Il s'agit ici de proposer une méthode expérimentale s'inspirant de la méthodologie Alceste pour identifier les formules rituelles dans un genre discursif particulier, rituel et épidictique, que sont les vœux des présidents de la Cinquième République. Nous montrons en quoi une exploration prenant comme unités les énoncés et non les formes permet d'identifier un certain nombre de figements et de travailler sur la variation de formules rituelles comme les marques de l'empathie, les formules d'adresses, les formules finales...
\end{abstract}

\section{ABSTRACT}

This paper propose an experimental method based on Alceste method in order to identify ritual forms in discourses of the fifth Republic Presidents' New Year speeches. We point out how an exploration based on utterances and not on forms permit to identify frozen structures and also to work on ritual formula variation as empathy mark, address, and conclusion...

\section{Les vœux présidentiels, un genre discursif empathique}

Le message du 31 décembre est constitutif d'un rituel institutionnel et politique où s'exprime le pouvoir du président élu par le peuple. Il symbolise à la fois la fonction de représentation du président de la République, sa relation au peuple, la personnalisation et la personnification du pouvoir présidentiel, la continuité et la pérennité des institutions étatiques. À l'exception des conférences de presse du 14 juillet, entrées plus tardivement dans les usages, abandonnées par Sarkozy puis

\footnotetext{
* Université Paris-Est Créteil, Céditec (EA 3119).
} 
reprises par Hollande en juillet 2012, les vœux sont les seuls rendezvous véritablement fixes du président avec ses concitoyens sous la Cinquième République.

Les messages de vœux des présidents français reposent sur des stratégies de communication qui combinent des usages obligés et des signes marqués, visant à gérer l'opinion publique, à faire face aux évènements et à établir de brefs bilans politiques. Car au-delà des actes, des évènements et d'une image construite en partie par les médias, c'est aussi à travers le discours que les dirigeants véhiculent une représentation confortant leur ethos ${ }^{1}$. S'appuyant sur un rituel bien établi, ces stratégies discursives sont un moyen, au-delà de l'action politique, de façonner par le discours l'Histoire nationale et la trace laissée par le dirigeant politique.

Ces discours éminemment politiques appartiennent à un genre discursif particulier, fortement codifié et relèvent du genre épidictique ${ }^{2}$. Dans ce cadre très contraint, le président de la République peut-il innover, se réapproprier certaines formules, proposer un nouveau mode d'expression des vœux?

Au cours de travaux précédents, nous avons mis en lumière un certain nombre de régularités tout en nous efforçant d'examiner, sinon l'ethos de ces locuteurs successifs, du moins des profils énonciatifs, parfois des styles. Nous avons également étudié la variation des formules rituelles sur l'ensemble de la période et, pour les dernières années du corpus, plus particulièrement cherché à voir comment les présidents de la République s'appropriaient ces formules rituelles et les adaptaient ou non à leur pratique discursive, au contexte évènementiel.

Nous avons montré en outre que les vœux présidentiels, après la période gaullienne, constituent un genre politique empathique. C'est cet aspect qui sera plus particulièrement analysé ici, plus que le caractère politique ou argumentatif, plus que l'aspect épidictique dont on trouve de nombreuses traces au sein d'énoncés valorisant les valeurs communes et magnifiant les valeurs de mérite et d'effort.

1. La notion d'ethos, retravaillée par l'analyse de discours, provient de la rhétorique aristotélicienne et désigne l'image de soi construite par le locuteur à travers son discours.

2. Parmi les genres rhétoriques, le genre épidictique désigne ce qui relève de l'éloge et du blâme, de l'apparat et de la célébration des valeurs communes. 


\section{Présentation du corpus}

Les analyses présentées portent sur l'ensemble des messages de vœux de la Cinquième République de 1959 à 2014. Dans le cadre d'une démarche expérimentale, ce corpus est soumis à variations. Les premières analyses portent sur une période s'étendant de 1959 à 2001 (de Gaulle, premier septennat de Chirac). Nous éprouverons le corpus complet à l'aune des formules identifiées sur le corpus des septennats (septennats + quinquennats, soit 1959-2014) [fig. 1, page suivante].

À côté du tableau présentant les caractéristiques quantitatives de ce - petit - corpus, l'analyse arborée de la connexion lexicale, calculée au moyen d'Hyperbase et ne tenant compte que de la présence-absence du vocabulaire permet de saisir les principales oppositions du corpus. Il s'agit donc dans ce premier mouvement de s'intéresser à l'ensemble des mots constituant notre corpus. La représentation arborée traduit ainsi les distances ou proximités entre les différents discours en termes d'emplois du «stock lexical». Il convient de noter que ce type de calcul produit des configurations plus tranchées que ne le ferait une arborée des distances qui tiendrait compte des fréquences ou une analyse factorielle des correspondances ${ }^{3}$ portant sur un tableau lexical et sur des effectifs absolus, mais le propos n'est pas celui de cette contribution.

Quels que soient les véritables auteurs de ces discours, les proximités entre les textes reconstruisent fidèlement les mandats successifs. Nous soulignerons la particularité relative des mandats Giscard/Pompidou qui se fondent en une même zone du «plan lexical», mais surtout la rupture qui semble se manifester après le premier mandat de Chirac ${ }^{4}$ et qui pourrait conduire à émettre l'hypothèse que les quinquennats ne présentent pas la même homogénéité que les septennats, Chirac (2), Sarkozy et Hollande n'étant pas aussi clairement identifiables que leurs prédécesseurs. Il n'y a pas de raison à priori pour que la longueur du mandat modifie fondamentalement l'exercice des vœux. Pourtant la statistique nous suggère une rupture que nous tentons d'expliquer dans un ouvrage à paraitre consacré aux vœux présidentiels.

3. Désormais AFC.

4. Ce phénomène est confirmé par d'autres analyses, notamment des AFC pratiquées sous Lexico 3 et TextObserver. 


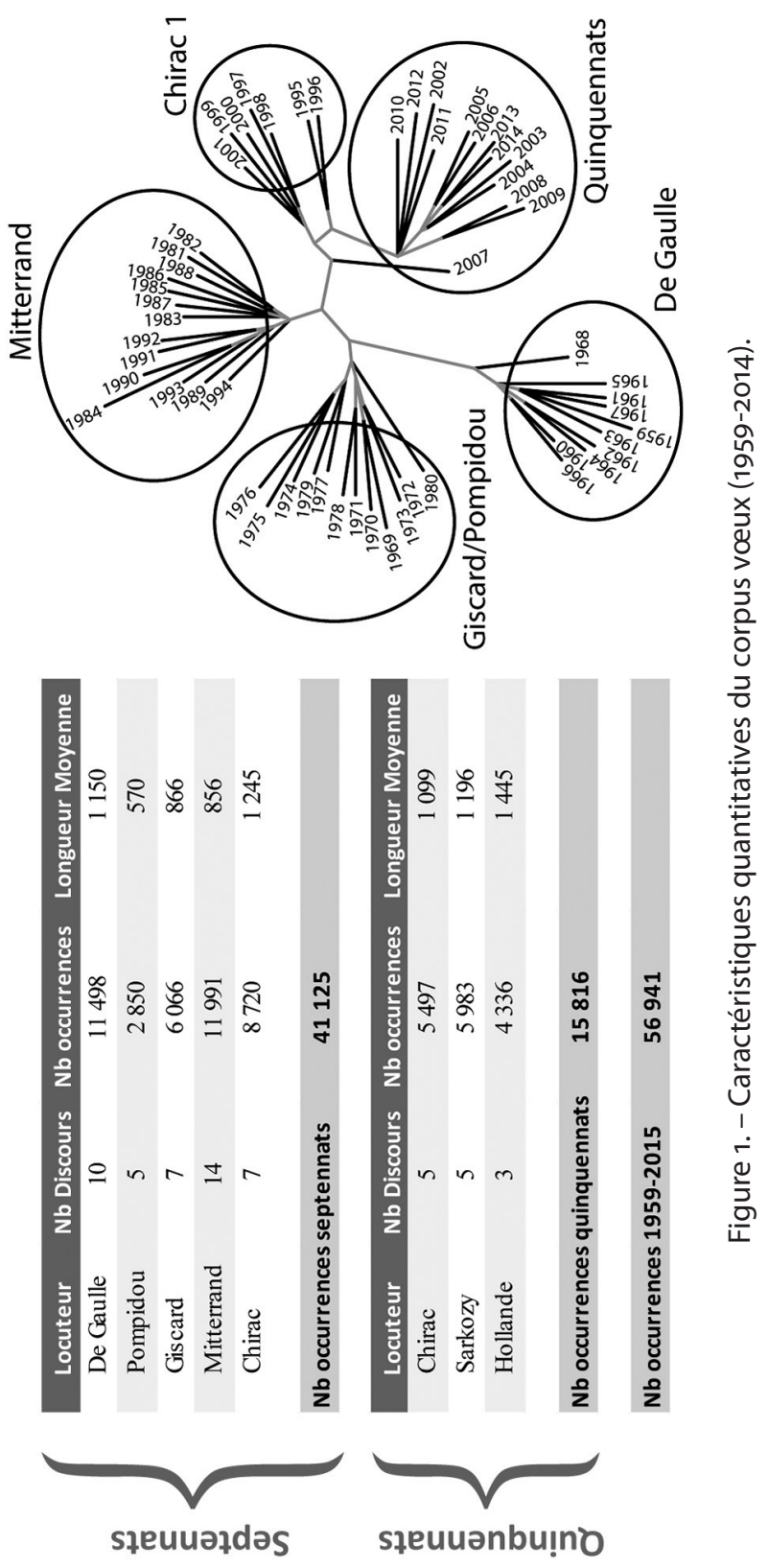




\section{La méthodologie Alceste, principes et méthode}

Nous avons utilisé le logiciel Alceste ${ }^{5}$ afin de proposer les éléments d'une typologie des messages de vœux dans une approche consistant à quantifier le corpus pour en extraire les structures signifiantes. L'algorithme d'Alceste repose essentiellement sur la classification descendante au sujet de laquelle il ne s'agit pas de revenir ici en détail ${ }^{6}$. Notons simplement que les méthodes de classifications automatiques s'appliquent à des tableaux à double entrée, et que la particularité d'Alceste réside dans son opposition fondamentale aux outils dits «classiques» précisément sur cette notion de tableau lexical. En effet, Alceste croise, dans un tableau modélisant le texte, les énoncés en lignes et les mots ou lexèmes en colonnes.

\subsection{Une démarche inductive}

L'algorithme d'Alceste ne repose donc pas sur une segmentation préétablie, mais constitue des classes, indépendamment des grandes divisions du corpus, dans une perspective différente de celle que d'autres outils (Lexico3, TXM, TextObserver, Hyperbase et bien d'autres) nous ont permis d'adopter par ailleurs. Cette démarche s'inscrit dans la continuité de l'approche de Benzécri $(1977,1981)$ et du courant de l'analyse des données : mettre au jour l'organisation interne du discours plutôt que de rendre compte des différences statistiques entre les textes du corpus, Benzécri s'inspirant lui-même du distributionnalisme harrissien pour qui l'analyse distributionnelle du discours fournit un certain nombre

5. Alceste (Analyse des lexèmes cooccurrents dans les énoncés simples du texte) a été conçu par Max Reinert avant d'être distribué par la société Images.

6. Dans l'étude accessible à cette adresse (<http://leblanc.jeanmarc.free.fr $>$ ), nous revenons plus largement sur la méthodologie Alceste. Voir aussi Reinert $(1990,1993,1999)$. Le principe d'Alceste revient à découper le corpus en fragments de phrases (les énoncés). Ces énoncés sont ensuite classés en fonction de la distribution de leur vocabulaire, en d'autres termes en fonction des mots qu'ils ont en commun. La classification descendante hiérarchique suppose que l'on commence par isoler les énoncés les plus discriminants. Ces énoncés forment une première classe. On réitère l'opération jusqu'à épuisement des possibilités. La classification descendante hiérarchique part de l'ensemble pour arriver au plus petit, contrairement à la classification ascendante hiérarchique qui part du plus petit pour former un tout. 
d'enseignements sur certaines lois externes impliquées dans la production du discours. Ainsi l'influence benzécriste et celle de Harris (1969) nous éclairent sur la philosophie du logiciel. Selon Benzécri, l'analyse distributionnelle consiste non pas à chercher le sens d'un texte mais à déterminer comment sont organisés les éléments qui le constituent, ce que Reinert (1993) reformule de la façon suivante : "Il s'agit, non pas de comparer les distributions statistiques des "mots" dans différents corpus, mais d'étudier la structure formelle de leurs cooccurrences dans les énoncés d'un corpus donné.»

\subsection{Les étapes du traitement}

Une première étape consiste à repérer les unités de contexte initiales (UCI), c'est-à-dire les divisions structurelles du corpus (locuteur, année...) puis à identifier les variables ${ }^{7}$ (ces variables correspondent dans notre exemple aux locuteurs et aux années et prennent la forme de mots étoilés).

Le traitement se poursuit par une opération de réduction, manière de lemmatisation qui regroupe sous une même entrée les formes correspondant aux différentes flexions d'un lemme. Cette lemmatisation quelque peu brutale s'apparente plutôt à une racinisation. Ainsi on considèrera «mang» au lieu de «manger».

Cette première étape génère le dictionnaire du corpus par la recension des formes entières et des formes réduites. On procède ensuite au découpage du corpus en unités de contexte, énoncés correspondant plus ou moins à la phrase ${ }^{8}$. C'est à partir de ces UCE (unités de contextes élémentaires) et des mots qu'elles contiennent que s'effectuent les classifications. Les énoncés sont classés selon leur proximité, en fonction de la distribution de leur vocabulaire, selon une procédure de classification descendante hiérarchique.

Une fois le corpus racinisé et découpé en unités de contexte, la classification descendante hiérarchique identifie des classes d'énoncés. Cette classification procède par itérations successives : le corpus est considéré comme un ensemble d'énoncés. La première phase consiste à chercher la partition en deux. Sur la plus grande des classes obtenues on répète l'opération et ce jusqu'à saturation des possibilités. On obtient ainsi des classes d'énoncés selon les formes qui les composent.

7. Nos variables seront les 5 locuteurs et les 42 divisions chronologiques.

8. Sur ce point, se reporter à Reinert et Fenoglio (1998). 


\section{Les mondes lexicaux du corpus vœux}

Cette approche de la méthodologie Alceste nous permet d'identifier les grandes classes thématico-sémantiques du corpus des vœux présidentiels (1959-2001) et d'y situer les variables chronologiques ou individuelles au moyen de ces mots étoilés, contributifs ou non des classes identifiées par Alceste. Plus encore que la taille de la classe obtenue, l'ordre de l'émergence est essentiel dans l'interprétation'.

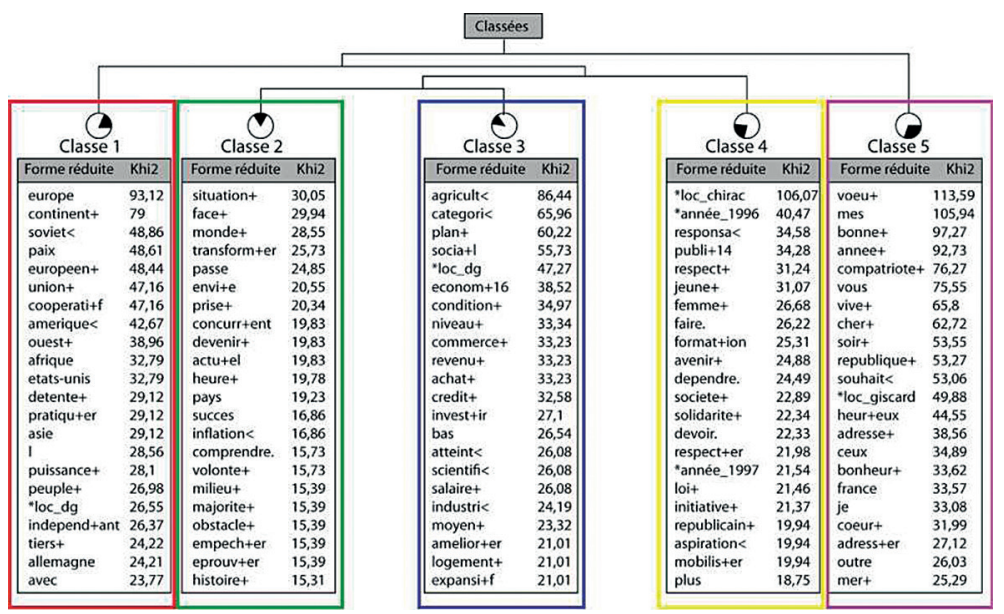

Figure 2. - Classification descendante hiérarchique du corpus vœux.

L'analyse retient 592 unités de contextes, soit $61 \%$ des énoncés composant le corpus. Ceci constitue une proportion courante compte tenu des expériences réalisées sur d'autres données. Parmi les énoncés classés, la classe 5 est la plus massive. Ce n'est pourtant pas là une évidence : la catégorie la plus discriminante, et donc la première à émerger du traitement, ne comporte pas nécessairement le plus grand nombre d'énoncés. La proportion des énoncés non classés, dont nous avons jugé qu'elle n'était pas inhabituelle au regard d'autres corpus que nous

9. Mots étoilés contributifs : on pourrait dire, pour aller vite, que, dans la classe 5, les énoncés composés des formes réduites, vœux, bonne, année, bonheur... sont particulièrement représentés chez Giscard. L'ordre d'émergence est important car l'algorithme d'Alceste identifie tout d'abord les énoncés qui sont les plus discriminés. Les énoncés rituels sont donc ceux qui se détachent dès la première phase de l'analyse. 
avons traités, représente les énoncés dont la cohérence n'est pas significative, et constitue le «résidu » du corpus, vu d'Alceste, résidu dont il convient de tenir compte lors de la phase interprétative : nos conclusions ne porteront que sur une partie du corpus. Cette classe 5, précisément parce qu'elle traite du rituel, nous intéresse tout particulièrement.

Les énoncés de la classe 5 (le rituel et les vœux) sont donc les plus discriminants. Apparaissent ensuite les classes 1 (politique internationale), puis 4 (valeurs démocratiques et républicaines) et enfin 2 (énoncés constatifs et bilans) et 3 (politique intérieure, économique et sociale). Bien entendu la nomination des classes ci-avant est déjà interprétative. Elle est le fait du chercheur et non du logiciel et est déduite de la liste des formes et du retour au texte.

\subsection{Une thématique du rituel}

La classe la plus importante quantitativement, qui couvre le lexique du rituel et des vœux et qui apparait donc comme le thème dominant de ce corpus, est aussi la première classe à se dégager, ce qui implique que les liens y sont les plus évidents entre les énoncés. C'est là où, de façon assez attendue, le taux de répétitivité est le plus fort. Les formes réduites qui la composent relèvent du lexique relatif aux vœux (vœu+, bonne+, année, souhait<, adresse, adresser), d'un lexique des formules d'adresse et du rituel (compatriote+, cher+, vive, France, république, outre mer), d'un champ lexical de l'affectif (bonheur+, cœur+, joie, famille+, ami+, aim+er, fet+er) et du champ lexical de la souffrance (solitude, malade+, maladie+, souffrir) ${ }^{10}$.

Si l'on s'en tient aux premières formes, la variable locuteur Giscard en est fortement constitutive, ce qui tend à confirmer des analyses menées précédemment, c'est-à-dire le caractère intimiste des messages mais aussi l'importance qu'il accorde aux vœux, au point que les aspects politiques et internationaux s'en trouvent parfois occultés. On se rappelle que Giscard ne semble pas bien se distinguer de Pompidou sur l'arbre. Pourtant, ce que l'on prête à Giscard ici ne s'applique pas particulièrement à Pompidou. Si leurs discours présentent des similitudes sur l'ensemble du vocabulaire, ils se distinguent néanmoins dans les emplois des formules rituelles.

10. Guide de lecture : les signes + indiquent qu'il peut s'agir du singulier et du pluriel. Les signes < signalent que souhait< renvoie à la fois à «souhaite, souhaitent, souhaiter, souhaitons...». 
Cependant, il ne s'agit pas de plaquer notre interprétation sur le contraste des locuteurs, selon la démarche qui est d'usage avec les outils visant à établir des distributions lexicales dans les sous-parties d'un corpus. On dira plutôt, si nous devions vulgariser la méthode, qu'au sein de la classe 5 figurent les formes comprises dans des énoncés relatifs au rituel et que ces énoncés sont pour une large part puisés chez Giscard, mais non exclusivement. Si l'on regarde de plus près, la forme compatriotes qui figure au cinquième rang de la classe, n'est pas attestée chez Giscard. Cette formule d'adresse n'a été adoptée qu'à partir de Mitterrand, ce que les spécificités, le retour au texte, pratiqués au moyen des outils longitudinaux, mais également le concordancier d'Alceste, permettent d'affirmer avec certitude.

Il convient également de noter qu'une forme peut apparaitre au sein de plusieurs classes ${ }^{11}$. Parmi les formes significatives, certaines paraissent inattendues. C'est le cas de la forme malades qui entre dans la réalisation des invitations des chefs d'État à se tourner vers «ceux qui souffrent». L'énoncé ci-dessous en fournit une bonne illustration ${ }^{12}$ :

Mes chers compatriotes, je suis heureux de vous dire, ce soir, les vœux très sincères que je forme pour vous et pour tous ceux que vous aimez.

Beaucoup d'entre vous sont en famille et s'apprêtent à fêter la nouvelle année. D'autres sont seuls, malades, ou dans la peine. $\left(\right.$ Chirac, 1997) ${ }^{13}$

Une analyse lexicométrique classique, que nous avons menée en comparant les énoncés de chaque classe, montre que le rituel est par ailleurs caractérisé par une forte représentation des marques personnelles : mes, mon, vous, je, mais aussi ceux. Les clés catégorielles indiquent que les catégories les plus significatives sont précisément les marqueurs de la personne ainsi que les démonstratifs et les indéfinis

11. Selon son environnement, une même forme peut apparaitre au sein de classes différentes. C'est le cas, par exemple, de la forme chomage, qui appartient à la fois au vocabulaire économique (lutter contre le chomage), mais qui apparait aussi dans la classe du rituel (je pense à ceux que touche le chomage). Il serait probablement judicieux d'envisager des procédures d'annotations contextuelles permettant de distinguer les différents emplois d'une même forme afin de la quantifier plus finement ensuite.

12. Les données complètes sont disponibles à l'adresse <http://leblanc.jeanmarc.free.fr $>$. On y trouvera notamment la totalité des énoncés de chaque classe identifiée.

13. En gras, les mots les plus contributifs. 
parmi lesquels le pronom démonstratif ceux. Une analyse et un retour au texte minutieux montre que la classe du rituel et des vœux s'articule selon les trois pôles énonciatifs je, vous, ils/ceux. Les quelques énoncés produits ci-dessus sont tout à fait significatifs des marques de la première du singulier et de la seconde du pluriel. Quant à la troisième du pluriel, eux, ceux, le référent est majoritairement «ceux d'entre nous», «ceux d'entre vous», «qui souffrent», «qui vivez dans la solitude»/ «la souffrance» / «les épreuves». Une expérience cooccurentielle portant sur ce pronom démonstratif place d'ailleurs les lexies relevant du paradigme de la souffrance parmi les premiers rangs. Les segments répétés comprenant cette forme pronominale montrent, par exemple, que sept segments attestent «ceux qui souffrent».

Nous avons, dans une analyse plus complète, quantifié la part du rituel chez chacun des locuteurs en isolant les seuls énoncés de la classe identifiée comme celle du rituel et en constituant un sous-corpus que nous avons soumis à Lexico $3^{14}$.

À titre d'exemple, le graphique qui suit est produit d'après les valeurs relatives en nombre d'occurrences. On a ramené les occurrences de la classe 5 à la taille des sous parties du corpus.

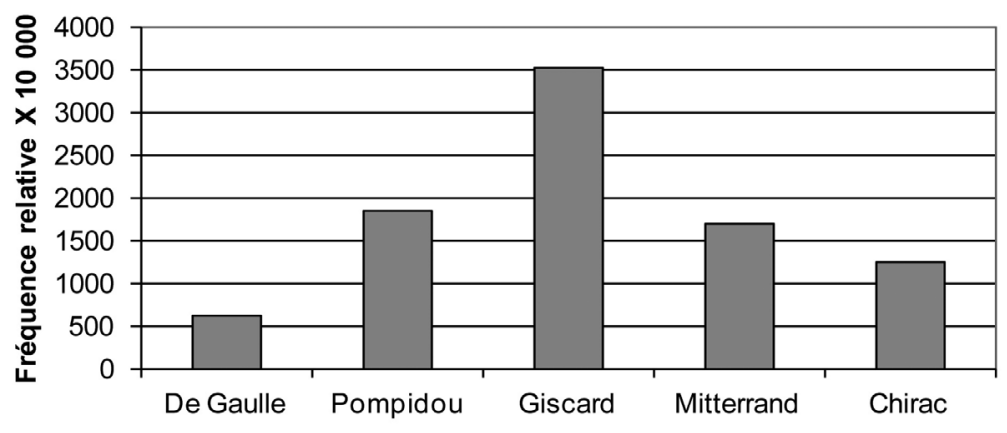

Figure 3. - Distribution des énoncés de la classe 5 en fréquences relatives.

14. Les indices de Khi2 portés sur les variables locuteur sont suffisants pour juger de la place accordée aux vœux par tel ou tel chef d'État. De même, Alceste permet de dénombrer le nombre d'énoncés entrant dans chaque classe. Notre expérience vise à quantifier en occurrences la part du rituel et non plus en énoncés, afin de croiser deux pratiques, mais surtout, la constitution de ce corpus expérimental soumis à Lexico 3 nous offre le moyen d'examiner les positionnements énonciatifs dans chaque classe thématique. 
Les fréquences relatives montrent qu'une large part du discours est accordée au rituel et aux vœux chez Giscard. La part des vœux chez Mitterrand est comparable au niveau observé chez Pompidou. On s'interrogera sur le cas du second président chez qui les énoncés sont souvent familiers mais qui pourtant ne semble pas accorder une place si grande aux vœux et au rituel. Les messages de Chirac sont caractérisés par une faible proportion d'énoncés relatifs aux vœux. L'examen des messages de Sarkozy et Hollande montre d'ailleurs une diminution sensible de la part accordée aux vœux.

\section{Caractéristiques du rituel : synthèse}

- La classe 5 est la première à émerger de la classification descendante.

- Elle est également la plus importante sur le plan quantitatif.

- Elle est essentiellement composée d'énoncés rituels.

- Giscard en est le plus constitutif.

- Les formes Je, Vous et Ceux en sont les pôles énonciatifs majeurs.

- Les clés catégorielles les plus significatives sont les marqueurs de la personne, les démonstratifs et les indéfinis.

- Selon la quantification opérée sous Lexico 3, la part du rituel se distribue ainsi dans l'ordre décroissant : Giscard, Pompidou, Mitterrand, Chirac, de Gaulle.

- Parmi les absences significatives : le pronom personnel de la troisième personne du singulier, la première du pluriel. Les variables d'auteur : de Gaulle, mais aussi Chirac. Les variables chronologiques (dans l'ordre décroissant) : 1963, 1960, 1995, 1996, 1964 et 1971. Parmi les modalités : falloir et devoir que l'on trouve plus particulièrement dans les autres classes construites à partir d'énoncés plus politiques. Enfin, les thèmes politiques et sociaux en sont rejetés : les racines econom, etat, europe, social en constituent la part essentielle.

- Les contrastes énonciatifs qui se manifestent sur le corpus global sont, dans les seuls énoncés rituels, fortement atténués.

\section{Analyse récursive du rituel et des vœux}

Nous proposons d'affiner la classification obtenue en utilisant Alceste de façon récursive, c'est-à-dire en soumettant les classes reconnues par cette première analyse à une série de traitements successifs. Nous nous en tiendrons ici à la seule classe du rituel.

La classification double établit 5 classes sur 61,8\% des énoncés. 
Lorsqu'on isole les seuls énoncés rituels pour les soumettre à un nouveau traitement, la thématique des vœux se décompose en plusieurs catégories d'énoncés dont nous produisons la synthèse ci-dessous.

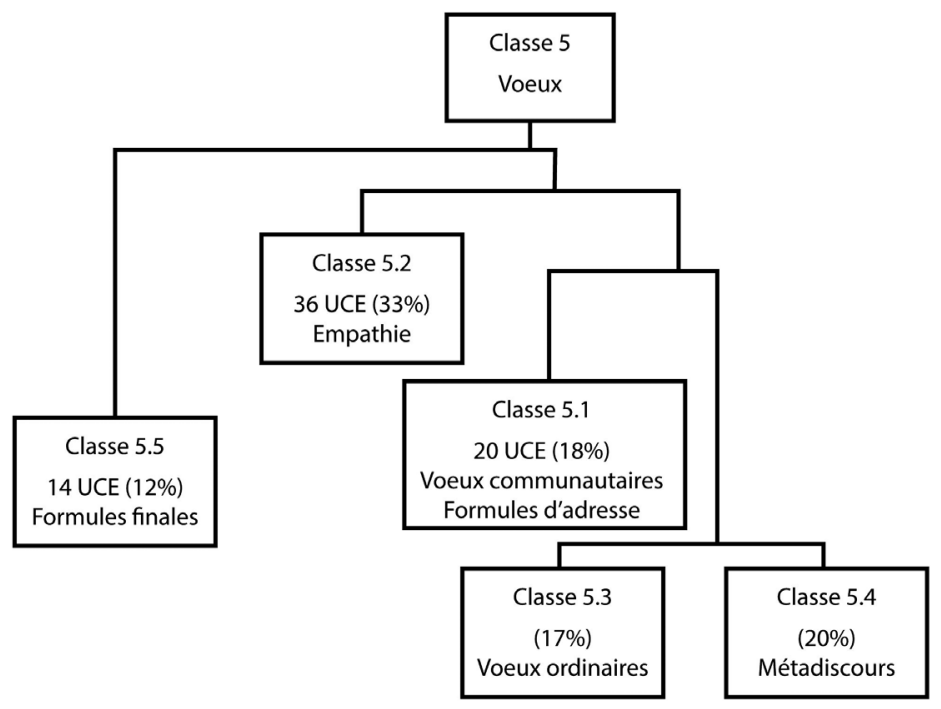

Figure 4. - Synthèse de l'analyse récursive des vœux.

\section{Énoncés les plus contributifs des sous-classes du rituel}

5.4. Ce soir, ce n'est pas le chef de l'État qui vient vous parler de politique. C'est l'un d'entre vous auquel vous avez confié démocratiquement, il y a trois ans et demi, la première responsabilité dans la République, qui vient vous exprimer ses voux. (VGE, 1977)

5.3. Françaises, Français, du fond de mon cœur, je souhaite que l'année 1974, malgré quelques bourrasques, vous apporte à chacune et à chacun de la joie dans votre vie personnelle, familiale, dans vos projets. (Pompidou, 1973)

5.2. Françaises, Français, avant de vous quitter, je-pense à celles et à ceux d'entre vous qui connaissent le deuil, les chagrins, le poids de la maladie et de la solitude, qui souffrent du chômage. (Mitterrand, 1981)

5.5. Vive la République Vive la France.

5.1. Oui, tous! C'est-à-dire, ceux de notre métropole, ceux de nos départements et territoires d'outre mer, ceux qui vivent à l'étranger enfin, cas très émouvant et qui nous est d'autant plus cher, ceux de la nation française au Canada. (De Gaulle, 1967) 


\subsection{Les formules finales, classe 5.5}

La classification descendante identifie la classe 5.5 en premier lieu; les énoncés qui la composent sont donc les plus discriminants. Celle-ci comporte $12 \%$ des énoncés classés, soit 14 unités de contexte. Il s'agit des formules finales (vive la République, vive la France) et de ces seules formules. Le tableau ci-dessous en présente les formes les plus significatives (vive + , republique +, france, et la variable de Gaulle sont les seules formes notées comme constitutives de cette classe).

\begin{tabular}{|lrccc|}
\hline Forme réduite & Khi2 & UCE cl & UCE tot & UCE cl \% \\
& & & & \\
vive+ & 112 & 14 & 14 & $100,00 \%$ \\
republique+ & 78,32 & 14 & 19 & $73,68 \%$ \\
france & 24,73 & 14 & 44 & $31,82 \%$ \\
*loc_dg & 20,57 & 7 & 14 & $50,00 \%$ \\
\hline
\end{tabular}

Tableau 1. - Formes significatives de la classe 5.5.

\subsection{Le paradigme de la souffrance, l'empathie présidentielle, classe 5.2}

La classe repérée ensuite par la classification (classe 5.2) représente $33 \%$ des UCE classées, soit 36 unités de contexte.

Les énoncés produits par le locuteur Mitterrand en sont particulièrement constitutifs (Mitterrand, 31,73 au premier rang des formes réduites de la classe). On y trouve cependant également Pompidou, Giscard et Chirac. Il est intéressant de constater que les énoncés significatifs de cette classe sont orientés vers la troisième du pluriel (ils, eux, ceux). On y trouve également des formules d'adresse (chers, compatriotes), le verbe penser (khi2 de 28) et un lexique important de la souffrance (maladie, malade, chomage, frapper, souffrir, épreuve, victime, pauvre, solitude, peine, cruel). Enfin, l'année 1987 est particulièrement remarquable, malgré un khi2 relativement faible (6). Cette perspective paradigmatique est confortée par les contextes. On constate ainsi que cette classe couvre l'essentiel des signes adressés en direction des Français, victimes d'évènements particuliers ou plus généralement les plus pauvres ou qui vivent des moments difficiles. Ainsi, la forme pens+er constitue le pivot de ces assertions. La charge sémantique du verbe ou du paradigme de la pensée est exclusivement orientée en ce sens. 


\begin{tabular}{|c|c|c|c|c|}
\hline Forme réduite & Khi2 & UCE cl & UCE tot & UCEcl \% \\
\hline * loc_mitterrand & 37,73 & 24 & 32 & $75,00 \%$ \\
\hline pens+er & 28,37 & 12 & 12 & $100,00 \%$ \\
\hline chert & 23,44 & 18 & 25 & $72,00 \%$ \\
\hline compatriote + & 19,44 & 18 & 27 & $66,67 \%$ \\
\hline ceux & 18,58 & 16 & 23 & $69,57 \%$ \\
\hline fetter & 15,76 & 7 & 7 & $100,00 \%$ \\
\hline qui & 15,49 & 24 & 45 & $53,33 \%$ \\
\hline maladie+ & 13,38 & 6 & 6 & $100,00 \%$ \\
\hline malade+ & 13,38 & 6 & 6 & $100,00 \%$ \\
\hline chom +23 & 11,05 & 5 & 5 & $100,00 \%$ \\
\hline soldat+ & 11,05 & 5 & 5 & $100,00 \%$ \\
\hline frapp+er & 11,05 & 5 & 5 & $100,00 \%$ \\
\hline ete & 11,05 & 5 & 5 & $100,00 \%$ \\
\hline aim+er & 11,05 & 5 & 5 & $100,00 \%$ \\
\hline ou & 10,95 & 14 & 23 & $60,87 \%$ \\
\hline mes & 10,77 & 18 & 33 & $54,55 \%$ \\
\hline souffrir. & 9,82 & 6 & 7 & $85,71 \%$ \\
\hline an + & 9,27 & 9 & 13 & $69,23 \%$ \\
\hline ont & 9,21 & 8 & 11 & $72,73 \%$ \\
\hline encore & 8,76 & 4 & 4 & $100,00 \%$ \\
\hline epreuve+ & 8,76 & 4 & 4 & $100,00 \%$ \\
\hline victime+ & 8,76 & 4 & 4 & $100,00 \%$ \\
\hline pauvre+ & 8,76 & 4 & 4 & $100,00 \%$ \\
\hline abord+ & 7,62 & 5 & 6 & $83,33 \%$ \\
\hline vers & 7,62 & 5 & 6 & $83,33 \%$ \\
\hline solitude+ & 7,26 & 6 & 8 & $75,00 \%$ \\
\hline peine + & 7,26 & 6 & 8 & $75,00 \%$ \\
\hline *année_1987 & 6,51 & 3 & 3 & $100,00 \%$ \\
\hline $\mathrm{cru}+\mathrm{el}$ & 6,51 & 3 & 3 & $100,00 \%$ \\
\hline nouvel+ & 5,55 & 7 & 11 & $63,64 \%$ \\
\hline $\mathrm{ma}$ & 5,5 & 4 & 5 & $80,00 \%$ \\
\hline fois & 5,5 & 4 & 5 & $80,00 \%$ \\
\hline jour+ & 5,5 & 4 & 5 & $80,00 \%$ \\
\hline simple+ & 5,5 & 4 & 5 & $80,00 \%$ \\
\hline seul+ & 5,35 & 6 & 9 & $66,67 \%$ \\
\hline famille+ & 4,58 & 8 & 14 & $57,14 \%$ \\
\hline aller. & 4,23 & 7 & 12 & $58,33 \%$ \\
\hline cette & 4,23 & 7 & 12 & $58,33 \%$ \\
\hline sentir. & 3,49 & 3 & 4 & $75,00 \%$ \\
\hline comme & 3,49 & 3 & 4 & $75,00 \%$ \\
\hline etes & 3,49 & 3 & 4 & $75,00 \%$ \\
\hline dans & 3,33 & 14 & 31 & $45,16 \%$ \\
\hline plus & 3,18 & 7 & 13 & $53,85 \%$ \\
\hline en & 2,89 & 13 & 29 & $44,83 \%$ \\
\hline nos & 2,34 & 7 & 14 & $50,00 \%$ \\
\hline mais & 2,14 & 4 & 7 & $57,14 \%$ \\
\hline avons & 2,14 & 4 & 7 & $57,14 \%$ \\
\hline meme & 2,14 & 4 & 7 & $57,14 \%$ \\
\hline autre+ & 2,14 & 4 & 7 & $57,14 \%$ \\
\hline je-pense & 2,14 & 4 & 7 & $57,14 \%$ \\
\hline pour & 2,14 & 17 & 42 & $40,48 \%$ \\
\hline pays & 2,14 & 4 & 7 & $57,14 \%$ \\
\hline
\end{tabular}

Tableau 2. - Les premières formes significatives de la classe 5.2. 
Ma pensée va à tous ceux qui ne pourront participer à la joie générale. (Pompidou, 1969)

Ayons une pensée pour tous, pour les victimes du Val d'Isère et du plateau d'Assy... (Pompidou, 1970)

Mais nous penserons d'abord à ceux qui souffrent, en ce jour, de solitude, de maladie, de pauvreté et de chagrins de toutes sortes. (Mitterrand, 1984)

Comment ne pas penser aux autres qui sont seuls et qui souffrent... (Mitterrand, 1985)

Mes chers compatriotes, à l'heure des væux de Nouvel An, vous trouverez normal que nous portions d'abord notre pensée vers nos soldats qui servent la France dans cette région du Golfe où pèse encore si lourd la menace de guerre. (Mitterrand, 1990)

Giscard est peu représenté. S'il accorde une large part à la thématique des vœux, il n'use que peu de l'empathie, du moins sous cette forme.

\subsection{Les vœux communautaires et les formules d'adresse, classe 5.1}

La classe 5.1 est la troisième à émerger du traitement. Elle représente $18 \%$ des unités de contexte classées soit $20 \mathrm{UCE}^{15}$. Nous produisons ci-dessous les énoncés les plus significatifs de cette classe.

Si Chirac en est le plus représentatif, l'examen des contextes montre que de Gaulle figure également dans cette classe. L'énoncé suivant (DG, 1967, khi2=29) est d'ailleurs le plus représentatif ${ }^{16}$ :

Oui, tous! C'est-à-dire, ceux de notre métropole, ceux de nos départements et territoires d'outre mer, ceux qui vivent à l'étranger enfin, cas très émouvant et qui nous est d'autant plus cher, ceux de la nation française au Canada. (De Gaulle, 1967)

Les autres énoncés répondent tous au même schéma. C'est la partie des vœux où le président s'adresse à tous les Français, les réunit en cette

15. On retrouvera le détail des données à l'adresse <http://leblanc.jeanmarc. free.fr/>.

16. Cet énoncé a le plus fort Khi2 de la classe, c'est-à-dire le coefficient d'appartenance à la classe le plus significatif. On comprend ainsi aisément le fonctionnement d'Alceste : tous les énoncés de la classe ont en commun les formes (réduites) métropole, outre mer, France, étranger... 
circonstance. C'est la partie des vœux qui assure la cohésion nationale. Il s'agit de formules d'adresse particulières ${ }^{17}$.

Les deux dernières catégories sont de fait les moins discriminantes. Il s'agit des classes 5.3 et 5.4.

\subsection{Les vœux "ordinaires", classe 5.3}

De la même façon que la classe précédente tendait à réunir les Français, la classe 5.3 groupe des énoncés à tournure plus familière. On ne présente plus ses vœux aux Français de la métropole, de l'étranger..., mais on formule des souhaits pour les Français, pour leur vie professionnelle, familiale, quotidienne. On pourrait évoquer ici un registre intime et familier, ce qu'on pourrait appeler les «vœux ordinaires », exprimés pour les Français, leur famille, leurs amis à qui l'on souhaite réussite et bonheur dans la vie professionnelle, familiale mais aussi santé.

Les énoncés de Pompidou en sont très constitutifs (loc_pompidou, khi2 de 37). On y trouve cependant également Giscard $(3,53)$ dans une faible mesure.

UCE $n^{\circ} 40$ Classe 3 Khi2=15 Françaises, Français, du fond de mon cour, je souhaite que l'année 1974, malgré quelques bourrasques, vous apporte à chacune et à chacun de la joie dans votre vie personnelle, familiale, dans vos projets. (Pompidou, 1973)

UCE $n^{\circ} 42$ Classe 3 Khi2=11 bonne année pour chacune de vous, bonne année pour chacun de vous. il est près de 8 heures et vous vous préparez sans doute à célébrer la fin de l'année avec votre famille, avec vos amis et peut-être aussi, quelques-uns, dans la solitude. (Giscard, 1974)

\subsection{Métadiscours et vœux : I'homme ordinaire et le président, classe 5.4}

Enfin, la classe 5.4 est très largement représentée par le locuteur Giscard (khi2 = 17,80). Il s'agit, là encore, de formuler des vœux dans une dimension plus volitive qu'attributive. On y note surtout la présence forte du statut présidentiel. Le plus souvent le président y revendique le statut

17. Les énoncés complets et les tableaux sont disponibles à l'adresse $<\mathrm{http}: / /$ leblanc.jeanmarc.free.fr/>. 
de l'homme ordinaire, c'est aussi l'expression du métadiscours sur les vœux. Les exemples qui suivent en fournissent l'illustration :

UCE $n^{o} 53$ Classe 4 Khi2 =30 lorsque les chefs d'État expriment leurs voux, on dirait qu'ils ne peuvent pas échapper à la règle de leurs fonctions et que leurs voux s'adressent inévitablement à la politique. (Giscard, 1975)

UCE $n^{\circ} 67$ Classe 4 Khi2 =14 ce soir, ce n'est pas le chef de l'État qui vient vous parler de politique. C'est l'un d'entre vous auquel vous avez confié démocratiquement, il y a trois ans et demi, la première responsabilité dans la République, qui vient vous exprimer ses vœux. (Giscard, 1977)

UCE $n^{\circ} 77$ Classe 4 Khi2 =12 C'est pourquoi les voux les meilleurs sont les plus simples. Je vous adresse les souhaits que les Français échangent entre eux depuis toujours : le bonheur, la santé et le succès. (Giscard, 1978)

\section{Analyse des formules empathiques}

Si nous isolons les seuls énoncés de la classe identifiée, comme représentant une certaine forme d'empathie présidentielle, pour soumettre ces données à un dernier traitement au moyen d'Alceste, deux classes émergent de l'analyse, retenant 94,5\% des unités, soit 35 UCE. Cette proportion importante s'explique aisément, les énoncés soumis à l'analyse étant très semblables.

Cette partition binaire est à considérer avec précaution mais révèle des faits intéressants. Elle traduit bien l'articulation des énoncés que nous avions observés. D'un côté, le lexique de la fête et du Nouvel An, des circonstances dans lesquelles se trouvent les acteurs de l'énoncé, locuteur et interlocuteur, au moment du discours. De l'autre, le lexique de la souffrance, souffrir, solitude, ceux, chomage. L'articulation apparait clairement classe 1: [Au moment où nous/vous apprêtons à fêter le Nouvel An, je pense, ou pensons], classe 2 : [à ceux qui souffrent, maladie, chomage, solitude...]. 


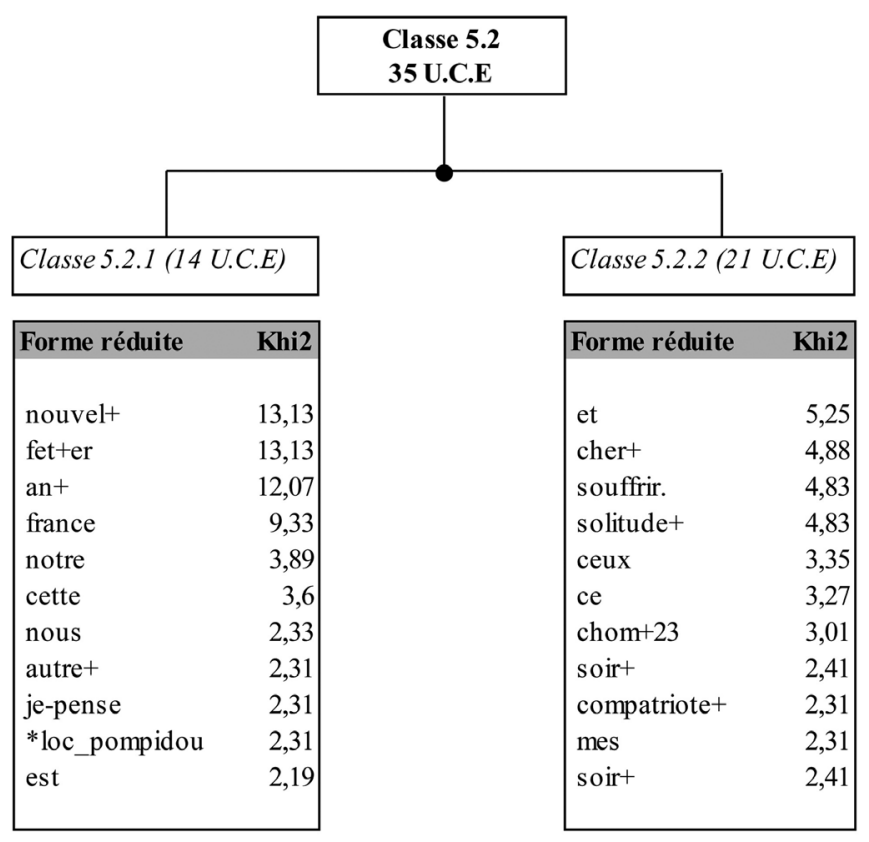

Figure 5. - Classes 5.2.1 et 5.2.2.

\section{Variation des formules rituelles et empathiques - corpus 1959-2014}

\subsection{Sarkozy à l'épreuve du rituel (2007, 2008)}

Les expérimentations menées au moyen d'Alceste ont permis d'identifier un certain nombre de régularités et de mettre au jour des éléments phraséologiques constitutifs du rituel discursif. À l'aune de ces résultats, nous avons analysé les deux premiers messages de Sarkozy, au sujet desquels les commentateurs politiques prédisaient une rupture avec les pratiques précédentes. Nous avons donc mené une série d'expérimentations afin d'examiner la façon dont Sarkozy se pliait, ou non, à l'exercice des vœux.

Parmi les verbes les plus fortement employés au sein des énoncés identifiés précédemment, la première personne du singulier et le verbe penser figurent parmi les premiers cooccurrents, chez tous les présidents de la République, de Gaulle excepté. Dans la quasi-totalité des emplois, le paradigme du verbe penser est utilisé pour exprimer un signe particulier en direction des Français les plus durement éprouvés. 
L'analyse approfondie du rituel montre que ces «pensées en direction des Français les plus démunis» ne sont pas nécessairement introduites par cette marque métadiscursive.

Et je voudrais en votre nom m'adresser ce soir aux plus malheureux des Français, à ceux pour lesquels ce soir ne sera pas une fête. Je veux dire d'abord les victimes des accidents cruels, et notamment les familles de ceux qui ont été victimes d'accidents du travail, comme celui qui a frappé la population minière du Nord. (VGE, 1974)

Je voudrais également adresser notre salut aux travailleurs immigrés qui vivent parmi nous, Portugais, Espagnols, Algériens, Marocains, citoyens des États francophones d'Afrique, qui nous apportent leur travail et leur activité, et qui doivent se sentir à leur place dans une société française qui les accueille. (VGE, 1974)

\subsection{Paradigme de la pensée}

Le paradigme de la pensée, à partir de Pompidou mais plus encore chez Mitterrand, est mobilisé essentiellement dans un signe en direction de «ceux qui souffrent» (Mitterrand, Chirac) ou vers «vous qui souffrez» (Sarkozy). On note en effet que si Sarkozy use bien de cette forme, il la dirige directement vers les Français «Vous qui souffrez» alors que ses prédécesseurs utilisent la troisième personne («pensons à ceux qui»). Il y a ici une appropriation de la formule rituelle. En 2008, Sarkozy revient à une tournure plus classique, ainsi qu'en témoignent les exemples qui suivent :

c'est la raison pour laquelle je veux penser d'abord à ceux que la vie a durement éprouvés, à ceux qui ont perdu leur emploi sans y être pour quoi que ce soit, à ceux qui sont victimes d'injustice, à ceux qui doivent affronter l'absence d'un être cher.

je veux penser à leurs familles qui vivent douloureusement cette séparation et plus encore à ceux qui pleurent un fils, un mari, un fiancé, un père.

Une rapide expérience permet de comparer Sarkozy à ses prédécesseurs sur l'emploi de ce paradigme de la pensée. Le groupe de formes qui suit se répartit ainsi sur l'ensemble du corpus en fréquences absolues :

- Pense : 29

- Pensée : 14

- Penser : 7

- Pensées : 4 
- Pensons : 3

- Pensais : 2

- Pensant : 2

- Penserez : 2

- Penserons : 1

L'histogramme des spécificités de ce groupe montre un net suremploi de la part de Sarkozy. On note, en outre, qu'il y a une affinité quantitative avec Giscard. De Gaulle, là encore, se démarque en sousutilisant nettement ce groupe des formes.

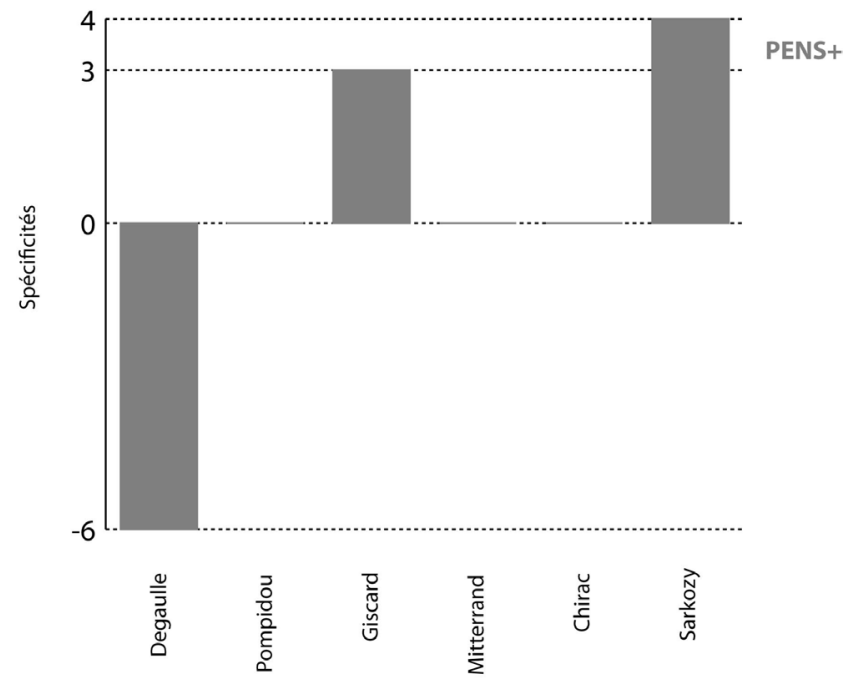

Figure 6. - Histogramme des spécificités du groupe de forme PENS+

Chez de Gaulle, il n'est aucunement question de s'intéresser à ceux qui souffrent. On note quelques emplois énumératifs chez Giscard, on remarque chez Mitterrand une proportion importante d'énoncés se rapportant à la thématique que nous recherchons $\mathrm{ici}^{18}$ («Nous penserons d'abord à ceux qui souffrent», «vous penserez ce soir à ceux qui souffrent et qui ont besoin d'amitié...»). Les cooccurrents spécifiques de ce groupe de formes permettent d'affiner quelque peu l'exploration textuelle des vœux présidentiels. On cherche ici à examiner quels mots ou quelles formes gravitent autour du groupe que nous avons constitué.

18. Pour l'analyse détaillée des occurrences de «penser», on se rapportera à l'étude en ligne mentionnée précédemment. 
En d'autres termes, dans un espace textuel donné (la phrase, le paragraphe, ou toute autre fenêtre contextuelle choisie) quels termes ont tendance à apparaitre dans les mêmes environnements que notre groupe de formes?

Le tableau qui suit produit les premières formes qui apparaissent le plus souvent au voisinage du paradigme de la pensée dans une fenêtre contextuelle représentant la phrase. Les mots cooccurrents du groupe «penser» sont donc, à l'exception des termes appartenant à ce groupe de formes, plus particulièrement des lexies renvoyant à la souffrance («souffrent», «maladie», «victimes», «deuil», «solitude», «inondations»... plus bas on trouve aussi le terme «chomage»), ou à la fête mais, dans ce cas, il s'agit d'emplois oppositifs («tandis que nous nous apprêtons à fêter le Nouvel An, pensons à ceux qui souffrent»*). Nous avons mis en évidence cette régularité dans l'analyse récursive du rituel au moyen de la méthodologie Alceste.

\begin{tabular}{|c|c|c|c|c|}
\hline Terme & Frq. Tot. & Frq. P. & Spécif & \multirow{37}{*}{$\begin{array}{l}\text { Guide de lecture: } \\
\text { Pour chaque terme le tableau } \\
\text { fournit sa fréquence totale, sa } \\
\text { fréquence partielle (fréquence } \\
\text { dans la partie considérée) et } \\
\text { l'indice de spécificité. Ainsi le } \\
\text { verbe «souffrent »apparaît } 12 \text { fois } \\
\text { au total, sur l'ensemble du corpus, } \\
\text { il apparaît } 7 \text { fois dans les phrases } \\
\text { où le groupe de forme (pense, } \\
\text { pensons, pensez...) est attesté. } \\
\text { Compte tenu des ces fréquences, } \\
\text { il y avait peu de chances, peu de } \\
\text { probabilités pour que ce verbe } \\
\text { apparaisse aussi souvent dans ces } \\
\text { phrases. Il est donc en sur-emploi. } \\
\text { Et c'est donc un cooccurrent } \\
\text { spécifique de ce groupe de } \\
\text { formes. Le premier terme apparaît } \\
\text { nécessairement. }\end{array}$} \\
\hline pense & 29 & 29 & 42 & \\
\hline pensée & 14 & 14 & 21 & \\
\hline ceux & 121 & 26 & 13 & \\
\hline penser & 7 & 7 & 11 & \\
\hline je & 411 & 41 & 8 & \\
\hline qui & 623 & 52 & 8 & \\
\hline souffrent & 12 & 7 & 8 & \\
\hline ou & 130 & 18 & 7 & \\
\hline maladie & 12 & 6 & 7 & \\
\hline pensées & 4 & 4 & 7 & \\
\hline victimes & 15 & 6 & 6 & \\
\hline solitude & 13 & 6 & 6 & \\
\hline soldats & 14 & 6 & 6 & \\
\hline vont & 15 & 5 & 5 & \\
\hline seuls & 16 & 5 & 5 & \\
\hline ma & 25 & 6 & 5 & \\
\hline pensons & 3 & 3 & 5 & \\
\hline celles & 25 & 6 & 5 & \\
\hline vers & 44 & 9 & 5 & \\
\hline fêter & 7 & 4 & 5 & \\
\hline connaissent & 3 & 3 & 5 & \\
\hline deuil & 3 & 3 & 5 & \\
\hline à & 1071 & 68 & 5 & \\
\hline vos & 47 & 9 & 5 & \\
\hline pensant & 2 & 2 & 4 & \\
\hline aussi & 116 & 12 & 4 & \\
\hline inondations & 2 & 2 & 4 & \\
\hline l'europe & 2 & 2 & 4 & \\
\hline pensais & 2 & 2 & 4 & \\
\hline injustice & 6 & 3 & 4 & \\
\hline soir & 48 & 7 & 4 & \\
\hline abord & 35 & 7 & 4 & \\
\hline soirée & 8 & 3 & 4 & \\
\hline nouvel & 20 & 5 & 4 & \\
\hline du & 341 & 25 & 4 & \\
\hline cruellement & 2 & 2 & 4 & \\
\hline
\end{tabular}

Figure 7. - Cooccurrents spécifiques du groupe PENS+. 
Nous conservons un certain nombre de formes (grisées) pour l'exploration qui suit. Ce groupe de formes que nous nommons «souffrance» se trouve en net sous-emploi chez de Gaulle et surreprésenté chez trois locuteurs : Mitterrand, Giscard et Sarkozy ainsi que le montre 1'histogramme de la figure 8. Cet histogramme revient à examiner, avec toutes les précautions qu'il convient d'observer, la part accordée par chacun des présidents au champ lexical de la souffrance, dont on sait, pour l'avoir analysé de façon approfondie, qu'il est constitutif du rituel. Si de Gaulle se démarque, là encore, très nettement, trois locuteurs présentent une distribution semblable de l'emploi de ce paradigme.

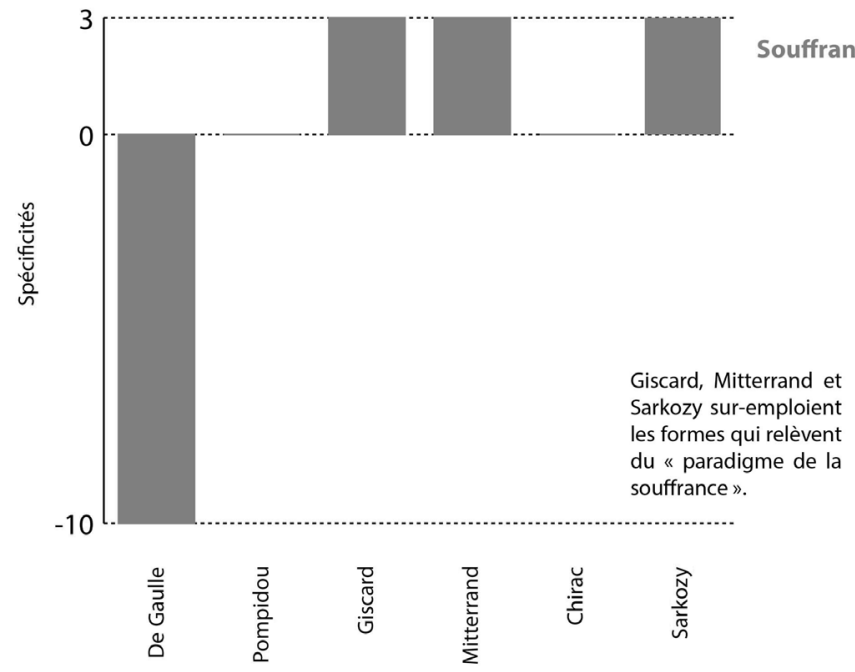

Figure 8. - Histogramme des spécificités du paradigme de la souffrance.

Voici quelques énoncés parmi les plus représentatifs de ce champ lexical :

ce soir, j'ai une pensée pour chacun d'entre vous. (Sarkozy, 2007)

je pense à vous qui êtes obligés de travailler cette nuit au service des autres et à vous, soldats français en opérations loin de vos foyers et qui risquez votre vie pour défendre nos valeurs. (Sarkozy, 2007)

je pense aussi à vous qui êtes seuls et pour qui cette soirée sans personne à qui parler sera une soirée de solitude semblable à toutes les autres. (Sarkozy, 2007)

je pense à vous, que la vie a éprouvés, et que la tristesse ou la douleur tiennent à l'écart de la fête. (Sarkozy, 2007) 
On notera que les vœux de Sarkozy en 2007 et 2008 réduisent la part du rituel et du lexique des vœux tout en conservant les éléments essentiels, constitutifs de l'ethos présidentiel (l'empathie, l'omniscience, le rôle de la France dans le monde).

\subsection{Formules rituelles empathiques 2009-2014}

La même expérience pratiquée sur le corpus 1959-2014 montre que les formules ne sont plus représentées aussi massivement.

Les tableaux qui suivent présentent en fréquence relative et en indice de spécificité la répartition des groupes de forme (souffrance et penser sur le corpus vœux 1959-2014).
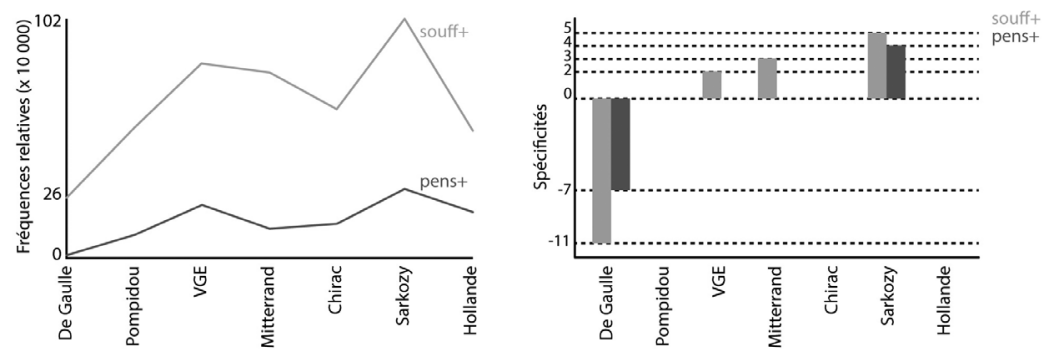

Figure 9. - Graphiques de fréquence relative et spécificités de souffrance et de penser.

Ainsi, les formules empathiques (en direction de ceux qui souffrent) ayant sensiblement évolué sur la fin de la période, la recherche d'empans textuels mobilisant de simples polycooccurrences de formes graphiques ne suffit plus à isoler les énoncés identifiés comme relevant de l'empathie présidentielle. En effet, dans les dernières années du corpus, le paradigme de la pensée se diversifie (certains emplois sont énumératifs dans des proportions plus importantes que dans la période 1959-2001). Par ailleurs cette empathie n'est plus, depuis 2008, exclusivement articulée autour du pronom «ceux».

Il conviendrait donc, si nous n'adoptions pas la procédure récursive sous Alceste, de procéder à une annotation qui permettrait d'isoler les lexies du paradigme de la souffrance et de procéder à des calculs polycooccurrenciels des formes ainsi annotées. Les énoncés qui suivent sont significatifs de ces reformulations : 
Sarkozy, 2011 :

Nous ne nous en sortirons pas en laissant de côté ceux qui souffrent déjà des conséquences douloureuses d'une crise dont ils ne sont pas responsables.

Hollande, 2012 :

J'exprime ma reconnaissance à l'égard de nos soldats pour leur courage, je salue la mémoire de ceux qui sont morts pour la France.

Hollande, 2012 :

Chers compatriotes, une de nos forces, c'est la solidarité. Nous la devons aux peuples opprimés. Mais nous la devons aussi surtout, auprès de nous, vers les plus fragiles, vers les malades, vers les personnes isolées, vers celles qui sont en situation de handicap, qui connaissent la précarité ou la solitude. Ce ne sont pas des assistés. [Noter le caractère polémique de cette formule empathique, ce qui n'est pas d'usage dans les messages précédents.]

\section{Cooccurrences multiples, segments répétés, inventaires distributionnels?}

La méthodologie Alceste et l'application expérimentale proposée ici permet de détecter des empans de façon plus large que nous ne le ferions à l'aide des segments répétés - ici sur la forme graphique - , qui permettent néanmoins d'identifier des figements tels que :

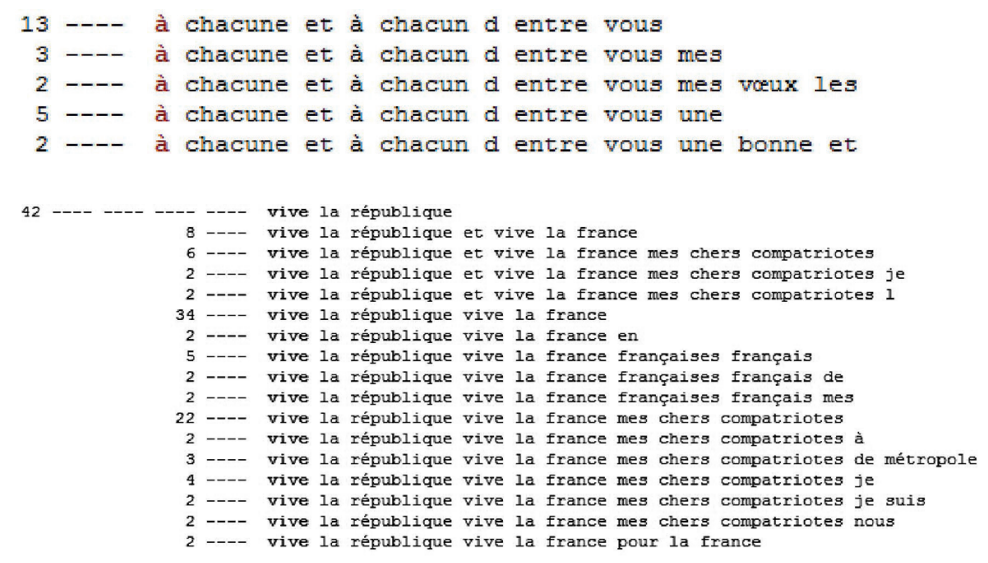

Notons que la fonctionnalité des inventaires distributionnels, implémentée de longue date dans Lexico 3, est une première réponse à l'étude de la phraséologie ou du moins un raffinement de la recherche des seg- 
ments répétés, notamment parce que ces inventaires montrent comment des syntagmes se distribuent à l'intérieur de segments plus longs.

Si les SR permettent en effet d'identifier des figements, ils sont insuffisants pour repérer les variations. C'est ainsi que l'on mobilise alors le plus souvent des calculs cooccurrentiels, voire polycooccurrentiels qui permettent d'extraire des empans textuels autour de formes pivots (Martinez \& Leblanc, 2005).

Une réponse envisageable à l'extraction d'empans textuels relevant de l'empathie présidentielle pourrait reposer sur l'utilisation de l'outil Unitex, développé par le laboratoire Linguistique Informatique Gaspard Monge (par, entre autres, Claude Martineau et Tita Kyriacopoulou).

Une première phase consisterait à construire un dictionnaire autour des lexies de la souffrance, une seconde reviendrait à construire des schémas syntaxiques permettant de modéliser les énoncés, tenant compte de la présence ou non du paradigme de la pensée (qu'il conviendrait d'annoter afin d'isoler les seuls emplois qui nous intéressent ici), du lemme «souffrir» pouvant être placé en début ou en fin d'énoncé et dont la présence est optionnelle, du pronom ceux, pouvant apparaitre ou non et d'une catégorie relevant du paradigme de la souffrance qu'il conviendrait de placer en début ou en fin d'énoncé.

Une modélisation complexe est nécessaire compte tenu des variations observées au sein de ces énoncés, variations que nous pouvons synthétiser de façon non exhaustive comme suit :

\begin{tabular}{|c|c|c|c|}
\hline $\begin{array}{l}\text { Je pense } \\
\text { Pensons } \\
\text { Ayons une pensée particulière } \\
\text { Tournons nous vers } \\
\text { Comment ne pas penser }\end{array}$ & $\begin{array}{l}\text { à vous quî } \\
\text { à ceux } \\
\text { aux millers de victimes } \\
\text { aux victimes }\end{array}$ & $\begin{array}{l}\text { connaissent } \\
\text { sont } \\
\text { souffrent }\end{array}$ & $\begin{array}{l}\text { la solitude } \\
\text { la maladie } \\
\text { qui travaillent } \\
\text { qui sont seuls } \\
\text { pour qui cette soirée n'est pas une fête } \\
\text { qui ont subi les innondations }\end{array}$ \\
\hline Nous la devons à & & & qui sont malades \\
\hline
\end{tabular}

On constate ainsi que, y compris sur des énoncés aussi codifiés que les plus codifiés des messages de vœux, la modélisation n'est pas des plus aisées et que la méthode Alceste permet de fournir des résultats probants ${ }^{19}$ : le principe de polycooccurrences entre les énoncés permet de s'affranchir de la position des formes dans la phrase au moment du

19. On trouvera à l'adresse suivante l'ensemble des énoncés de la classes relevant de l'empathie présidentielle : <http://leblanc.jeanmarc.free.fr/Chapitre4/ Analyse_recursive_Alceste/sur_voeux/UCE52.htm $>$. 
recueil des énoncés. La racinisation permet de faire le lien entre des énoncés comportant des formes réduites communes (p. ex. liberté, libérer, libération) qui se seraient trouvés isolés si le calcul avait porté sur la forme graphique. La classification ascendante permet de rapprocher des énoncés qui comportent beaucoup de formes communes, mais permet également de rendre certaines de ces formes optionnelles (penser, solitude, malade, maladie n'apparaissent pas dans tous les énoncés). Le coefficient de corrélation permet d'exclure les énoncés non pertinents. Dans une phase d'identification, la méthode Alceste semble donc la plus appropriée ${ }^{20}$. L'analyse des variations peut ensuite être menée au moyen d'autres outils ${ }^{21}$.

La recherche de motifs par expressions régulières sur un corpus étiqueté peut produire des résultats intéressants mais limités. L'expression suivante :

$[$ lemme $=\ll$ penser» $][]\{1 ; 20\}[$ lemme $=\ll$ souffrir $»]$ ou

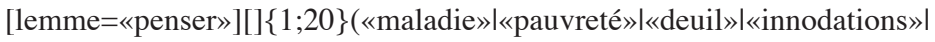
«chômage»)

ne permettent pas d'extraire la totalité des énoncés.

En tout état de cause, nous suggérons que l'étude de ces énoncés gagne par l'utilisation conjointe d'un outil mobilisant la méthodologie Alceste, pour le repérage des énoncés, et d'un outil longitudinal permettant la recherche de motifs complexes pour la quantification et le retour au texte. L'approche proposée, utilisant Alceste de façon récursive, nous semble répondre à la problématique des figements.

\section{Conclusion}

Si nous n'avons pas abordé de façon spécifique les problèmes de phraséologie ou de routines discursives, nous avons montré en quoi une exploration prenant comme unité les énoncés (méthodologie Alceste puis analyse récursive) et non uniquement les formes lexicales permet

20. Une fonctionnalité intéressante développée par Martinez et Leblanc (2005) (contextes spécifiques) permettrait également d'isoler certains de ces énoncés, une fois qu'ils auraient été identifiés par Alceste.

21. Coocs, Le Trameur, Alceste reposent finalement sur le même principe, celui des cooccurrences multiples, mais la démarche Alceste est inductive alors que Le Trameur ou Coocs supposent que l'on recherche une forme pôle dont on calculera ensuite les cooccurrents et polycooccurrents. 
de mettre en lumière un certain nombre de phénomènes de figements, de repérer des régularités mais aussi d'examiner la variation de formules rituelles relevant le plus souvent ici de l'empathie présidentielle.

L'expérimentation proposée - en particulier l'analyse récursive nous parait apte à enrichir les travaux traitant des phénomènes de phraséologie en portant sur les explorations mobilisant les inventaires distributionnels, les segments répétés et la recherche de cooccurrences multiples un éclairage complémentaire.

\section{RÉFÉRENCES BIBLIOGRAPHIQUES}

BENZÉCRI, Jean-Paul. (1977). Analyse discriminante et analyse factorielle. Les Cahiers de l'analyse des données, 4(2), 369-406.

Benzécri, Jean-Paul. (1981). Pratique de l'analyse des données. Paris : Dunod.

BENZÉCRI, Jean-Paul. (1982). Histoire et préhistoire de l'analyse des données. Paris : Dunod.

Charaudeau, Patrick \& Maingueneau, Dominique. (2002). Dictionnaire d'analyse du discours. Paris : Seuil.

Fiala, Pierre. (1987). Pour une approche discursive de la phraséologie. Remarques en vrac sur la locutionalité et quelques points de vue qui s'y rapportent, sans doute. Langage et société, 42, 27-44.

HARris, Zellig. (1969). Analyse du discours (trad. française). Langages, 13, $8-45$.

Martinez, William \& Leblanc, Jean-Marc. (2005). Positionnements énonciatifs dans les vœux présidentiels sous la Cinquième République. Analyse des marques personnelles par les méthodes de cooccurrence. Revue Corpus, 4. Disponible en ligne sur <http://corpus.revues.org/ 347> (consulté le 31 janvier 2016).

Reinert, Max. (1990). Système Alceste : une méthodologie d'analyse des données textuelles. Dans Actes des Journées internationales d'analyse statistique des données textuelles - JADT 1990 (p. 144-161). Barcelone.

ReInert, Max. (1993). Les «mondes lexicaux» et leur logique. Langage et société, 66, 5-39.

REINERT, Max. (1999). Quelques interrogations à propos de l'«objet» d'une analyse de discours de type statistique et de la réponse «Alceste». Langage et société, 90, 57-70.

Reinert, Max \& Fenoglio, Irène. (1998). Application d'Alceste à un corpus d'entretiens : de l'analyse de données à l'analyse de l'énonciation. Dans Actes des Journées internationales d'analyse statistique des données textuelles - JADT 1998. Nice. 